\title{
Modelling and Prediction of Photovoltaic Power Output Using Artificial Neural Networks
}

\author{
Aminmohammad Saberian, H. Hizam, M. A. M. Radzi, \\ M. Z. A. Ab Kadir, and Maryam Mirzaei \\ Department of Electrical and Electronic Engineering, Universiti Putra Malaysia (UPM), 43400 Serdang, Malaysia
}

Correspondence should be addressed to Aminmohammad Saberian; saberian.res@gmail.com

Received 9 January 2014; Accepted 26 February 2014; Published 6 April 2014

Academic Editor: Niyaz Mohammad Mahmoodi

Copyright (C) 2014 Aminmohammad Saberian et al. This is an open access article distributed under the Creative Commons Attribution License, which permits unrestricted use, distribution, and reproduction in any medium, provided the original work is properly cited.

\begin{abstract}
This paper presents a solar power modelling method using artificial neural networks (ANNs). Two neural network structures, namely, general regression neural network (GRNN) feedforward back propagation (FFBP), have been used to model a photovoltaic panel output power and approximate the generated power. Both neural networks have four inputs and one output. The inputs are maximum temperature, minimum temperature, mean temperature, and irradiance; the output is the power. The data used in this paper started from January 1, 2006, until December 31, 2010. The five years of data were split into two parts: 2006-2008 and 20092010; the first part was used for training and the second part was used for testing the neural networks. A mathematical equation is used to estimate the generated power. At the end, both of these networks have shown good modelling performance; however, FFBP has shown a better performance comparing with GRNN.
\end{abstract}

\section{Introduction}

The sun is one of the primary sources of energy for most of the natural processes such as heat, wind, and rain. The most effective conversion of sunlight into chemical energy can be seen in photosynthesis procedure. Currently human kind is using fossil fuels as their primary energy source but the fossil fuels are not an appropriate source of energy. This leads to the usage of sunlight as a renewable source of energy to produce electricity, called solar power. Moreover, solar energy is green energy and it is environmentally friendly. Photovoltaic panel and solar thermal are the main sources for electricity generation from solar energy. The prediction of power generation from solar energy is gaining considerable attention due to the increment of power generation from solar energy [1]. Numerical models have been developed to forecast weather and solar power; however, they require a powerful computing system $[2,3]$.

An artificial neural network assimilates human brain learning system and is able to find an input-output relationship for linear and nonlinear systems with less computational effort. This leads to the wide usage of artificial neural networks to forecast various criteria such as irradiance and temperature $[4,5]$. In general, neural networks are universal approximators [6]. The original of ANN was introduced by McCulloch and Pitts [7]. Although there has been research into the prediction of some parameters such as temperature and solar radiation, there has not been comprehensive research into the power prediction. A forecasting method using ANN has been proposed for solar radiation in [8]. The power generation of photovoltaic panels depends on solar radiation, temperature, humidity, and so on [9-13]. These parameters are naturally variable and nonschedulable; thus, the amount of generated power is generally unknown. Feedforward back propagation (FFBP) and the general regression neural network (GRNN) have been affirmed as two effective methods in modelling and prediction by previous researchers [14]. These two models are employed for the prediction of PV output power in this research since they have shown their effectiveness compared to statistical and autoregression approaches. [15]. PV plant modelling has become an active research field in the last few years, with the development 
of new models based on artificial intelligence techniques [16]. Also, solar radiation forecasting methods are presented in $[4,17]$ and a method for solar power forecasting is discussed in [18]. On the other hand, power planning is necessary for cost efficiency of power generation in which power forecasting is an essential part $[14,19]$. Some data is required in order to predict a solar panel generated power. However, sometimes such data is not available due to the lack of related databases [8]. Therefore, forecasting is required for sizing and meteorological data prediction $[19,20]$ to achieve an increase in the efficiency [21] and to schedule the operation of the system [18]. The artificial neural network (ANN) is widely used for solar data prediction in previous research work. AbdulAzeez [17] evaluated an artificial neural network for global solar radiation in Nigeria. Chen et al. [18] improved the neural network to forecast the power of PV panels. They mentioned that this method is accurate and efficient in the operation of a PV system. Linares-Rodríguez et al. [20] explained a multilayer perceptron feedforward neural network. These authors applied a trial process to find a suitable number of hidden layers for the prediction of daily global solar radiation. They concluded that although there are small changes in the results for a different number of hidden layers, the cost of computing is different and 25 neurons in the hidden layers is assumed as the best number. Paoli et al. [21] used a MLP to develop a methodology for solar radiation prediction. The author emphasized that the ability of an ANN for prediction is high. The application of ANN has some advantages such as the following: the mapping function in neural network is very flexible and neural network have the ability of generalizing form a limit set of data points and giving a good results at new data points [22]. Only a few describe forecasting models used to predict directly the daily energy production of the PV plant [23].

\section{Background of Research}

ANN $[18,21]$ and its related models such as FFBP $[4,18,19,24]$ and GRNN [8] are shown to be popular methods among authors. ANN is a model based on interconnected neurons to create an output by processing information based on the input. The relationship between the input and the output is based on the weight of the neurons and the input data for which the neurons have to be trained to the system [8]. Naini [25] discussed the application of three different neural networks structures such as radial basis function network, FFBP, and GRNN for prediction of the characteristics of the scour hole geometry. Vigneswaran and Dhivya [26] applied five accurate neural network models including FFBP and GRNN and concluded that GRNN has better results. The work discussed in [27] considered wind speed forecasting by using FFBP. In another work [28], the authors used FFBP to predict the velocity of crude oil. In [29] the authors applied FFBP for temperature forecasting and concluded that this model had the proper potential for complex modelling of the relation between various factors. The authors in [30] mentioned that FFBP is the best model of neural network for real-time forecasting due to least training time and fast

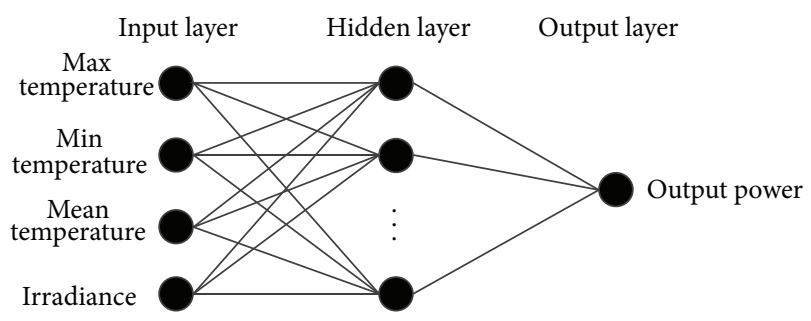

FIGURE 1: Diagram of prediction using FFBP.

response. In [31] the authors stated that FFBP is one of the most popular configurations of an ANN. The authors in [14] undertook load forecasting using FFBP. Zhao et al. [32] employed GRNN as one of the models employed to forecast wind power density. Some researchers have used many types of ANN for comparison and analysis of issues related to solar panels. Khatib et al. [8] applied four types of ANN for forecasting solar radiation forecasting. Their research was based on GRNN, FFNN, cascade forward back propagation neural network (CFNN), and Elman back propagation (ELMNN) scheme. Based on their research, the GRNN is better than the other models with a high level of accuracy and efficiency. One of the major advantages of a GRNN is defined as the effective relation of the model and the input [33].

\section{Artificial Neural Network}

3.1. Feedforward Back Propagation (FFBP). Among the different forms of ANN, feedforward back propagation and general regression neural networks are proposed in this research. A feedforward neural network is defined as a simple type of neural network in which the information flow is in the forward direction. Corani [34] utilized a feedforward neural network (FFNN) for air prediction in Milan. He described the structure of the FFNN in different three layers. An input layer was used for data collection, a hidden layer for data processing by neurons and the output layer for the results. Back propagation (BP) is assumed as a popular learning technique for feedforward [12] neural networks. Therefore the FFBP is defined as a model for forecasting parameters and the diagram of prediction using FFBP shown in Figure 1.

The FFNN is defined as a type of MLP network with special input values. These values that are multiplied by their weight are led to the hidden layer by neurons. In separate work, Khatib et al. [35] developed an artificial neural network for solar prediction in Malaysia. He provided a feedforward multilayer perception model with four inputs for the calculation. The training process for the back propagation algorithm includes a few steps. First, the weights are initialized for the neurons and the measured output is compared with the desired value to calculate the error. In the second step the weights are changed based on the error. In this way, the error is propagated backward to update the weights of the previous layers [19]. Kumari et al. [15] used a neural network for temperature prediction in India. 


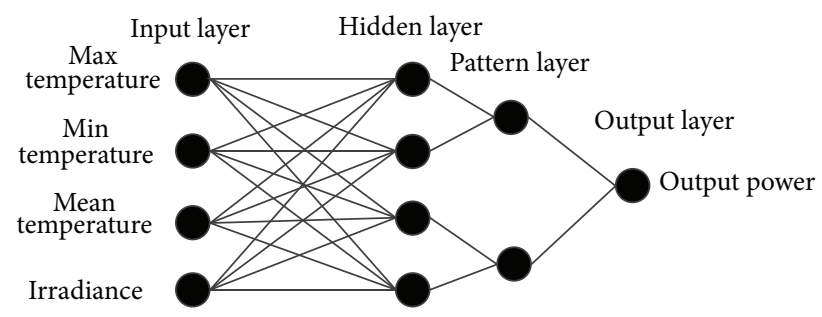

FIGURE 2: Architecture of GRNN.
TABLE 1: Cell open circuit voltage and maximum power point voltage at different irradiance.

\begin{tabular}{lccc}
\hline $\operatorname{Irr}\left(\mathrm{W} / \mathrm{m}^{2}\right)$ & $V_{\mathrm{oc}}(\mathrm{v})$ & $V_{\mathrm{mpp}}(\mathrm{v})$ & $V_{\mathrm{mpp}} / V_{\mathrm{oc}}$ \\
\hline 1000 & 30 & 23.8 & 0.793 \\
800 & 29 & 22.9 & 0.789 \\
600 & 28.56 & 22.27 & 0.78 \\
400 & 27.75 & 21.6 & 0.778 \\
200 & 27 & 20.8 & 0.77 \\
\hline
\end{tabular}

other words, the day length (DL) is required to be substituted by $h$ in the calculation where DL can be calculated as [8]

$$
\mathrm{DL}=\frac{2}{15} \cos ^{-1}(-\tan l * \tan \delta)
$$
can be forecasted by using a MLP neural network. Ahmed and Adam [24] explored a prediction model for solar radiation by use of an artificial neural network. The ANN in that study was a feedforward back propagation model. The results proved that this method is precise and suitable for forecasting solar radiation.

3.2. General Regression Neural Network (GRNN). A GRNN is defined as a probabilistic neural network that is definitely useful for prediction and performance comparison. GRNN is a new kind of neural network that Specht put forward in 1991 [36]. The network of a GRNN is the same as an FFNN with input, hidden, and output layers. The values from the hidden layer are led to a pattern layer. The pattern layer works as a summation layer with denominator summation and numerator summation neurons. The values of the hidden neurons are added by the denominator summation part and the values from the multiplication of the weights of the actual values of the hidden neurons are added by the numerator summation part. The output will be the division of the values from these two parts as shown in Figure 2.

\section{Methodology}

Meteorological data such as the temperature and solar irradiance were collected from the Malaysian Meteorological Department (MMD) that includes the daily minimum, mean average, and maximum temperature and solar irradiance starting from January 1, 2006, to December 31, 2010. All data was collected from the Kuala Lumpur International Airport (KLIA) station and the sample data for modelling of PV is shown in Table 4. However, a solar panel was not placed at this center and therefore the generated power data is not available. The solar irradiance measured is presented in units of $\mathrm{MJ} / \mathrm{m}^{2}$. The most commonly used unit for solar irradiation in photovoltaic system calculations is $\mathrm{W} / \mathrm{m}^{2}$. Thus, these data are converted according to the following equation:

$$
\frac{\mathrm{MJ}}{\mathrm{m}^{2}} \times \frac{1000000}{60 \times 60}=\frac{\mathrm{Wh}}{\mathrm{m}^{2}} \text {. }
$$

In order to convert the $\mathrm{Wh} / \mathrm{m}^{2}$ to $\mathrm{W} / \mathrm{m}^{2}$ the data values should be divided by the hours $(h)$. In this case $h$ is considered to be the time in which the solar irradiance is available. In where $l$ is the latitude and $\delta$ is defined as the declination angle. The value $\delta$ is given by [8]

$$
\delta=23.45 \sin \left[\frac{360(284+N)}{365}\right] .
$$

In (3), $N$ is the number of days and for the first day of January $(N=1)$, the solar day is considered to be 284 .

The next step is to calculate the two important parameters of solar panel which are the open circuit voltage $\left(V_{\mathrm{oc}}\right)$ and the short circuit current [37]. Both of these essential parameters are a function of the solar irradiance and temperature. However, in the datasheet of the solar panel [38] which is used in this research, the $V_{\mathrm{oc}}$ and $I_{\mathrm{sc}}$ are given at two certain points of (Irradiance $=1000 \mathrm{~W} / \mathrm{m}^{2}$, Temperature $=$ $\left.25^{\circ} \mathrm{C}\right)$ and (Irradiance $=800 \mathrm{~W} / \mathrm{m}^{2}$, Temperature $=20^{\circ} \mathrm{C}$ ). However, in modelling calculations $V_{\mathrm{oc}}$ and $I_{\mathrm{sc}}$ at different irradiance and temperatures are needed. In other words, the mathematical relation between these two parameters and the environmental factors of temperature and irradiance is required for solar panel modelling.

Accordingly the maximum power point voltage at the operating temperature and irradiance is calculated as follows:

$$
V_{\text {mpp }}=\left(\frac{V_{\mathrm{oc}} \times N_{T}}{100}\right) \times 0.78,
$$

where $V_{\text {mpp }}$ is the voltage at the maximum power point and $N_{T}$ is the normal thermal coefficient.

The value of 0.78 is an estimated coefficient. It is estimated that the maximum power point voltage can be considered as 0.78 of the open circuit voltage at a different working point. This value is the average ratio of $V_{\text {mpp }}$ to the $V_{\text {oc }}$ that is calculated based on the available data in the cell data sheet. Table 1 shows the cell open circuit voltage and maximum power point voltage at different irradiance.

Finally, the power can be estimated as in the following equation:

$$
P=V_{\text {mpp }} \times I^{2} .
$$

Therefore the power for the minimum, maximum, and mean temperature can be estimated based on (5). 


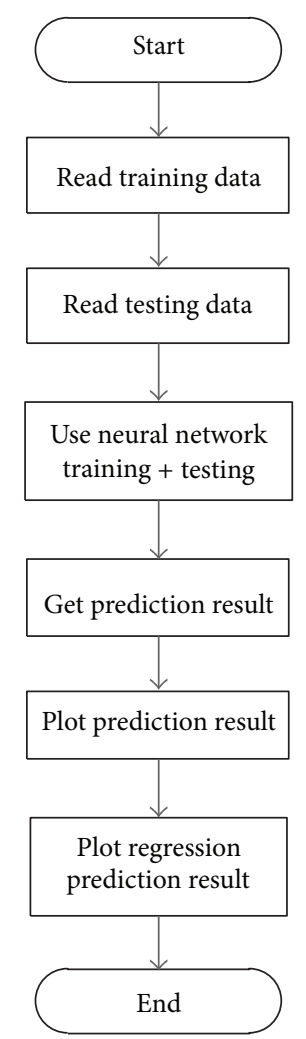

FIGURE 3: Flowchart of FFBP method.

4.1. FFBP Method. The feedforward back propagation method is used as a first neural network model for this study. The applied flowchart of FFBP is shown in Figure 3.

As shown in Figure 3, the prediction results are based on reading the testing and training data and using FFBP.

Before the training process, all the data must be normalized. Various normalization methods are used for neural networks to increase the reliability of the trained network [39]. The Min-Max normalization is applied in this research as follows:

$$
a=\left(b-b_{\min }\right)\left[\frac{a_{\max }-a_{\min }}{b_{\max }-b_{\min }}\right]+a_{\min },
$$

where $a$ is the normalized value and $b$ is a nonnormalized value. For this work, $a_{\max }=1$ and $a_{\min }=-1$.

After obtaining the result, the normalized values must become normal by using the following formula:

$$
b=\left(a-a_{\min }\right)\left[\frac{b_{\max }-b_{\min }}{a_{\max }-a_{\min }}\right]+b_{\min } .
$$

The mean squared error (MSE) is used for evaluation of predictive power as follows [15]:

$$
\mathrm{MSE}=\frac{1}{N} \sum_{t=1}^{N}\left(Z_{t}-\overline{Z_{t}}\right)^{2}
$$

where $\bar{Z}_{t}$ is a vector of the $N$ prediction and $Z_{t}$ is the vector of the real values.
TABLE 2: MSE for FFBP.

\begin{tabular}{lccc}
\hline $\begin{array}{l}\text { Number of neurons in } \\
\text { hidden layer }\end{array}$ & MSE train & MSE test & Regression \\
\hline 3 & 0.00019 & 0.000232 & 0.999157 \\
4 & 0.000322 & 0.000278 & 0.999005 \\
5 & 0.000364 & 0.000343 & 0.998773 \\
6 & 0.000155 & 0.000156 & 0.999427 \\
7 & 0.000164 & 0.000187 & 0.999328 \\
8 & 0.000136 & 0.000162 & 0.999405 \\
9 & 0.000137 & 0.000162 & 0.999408 \\
10 & 0.000104 & 0.000141 & 0.999482 \\
\hline
\end{tabular}

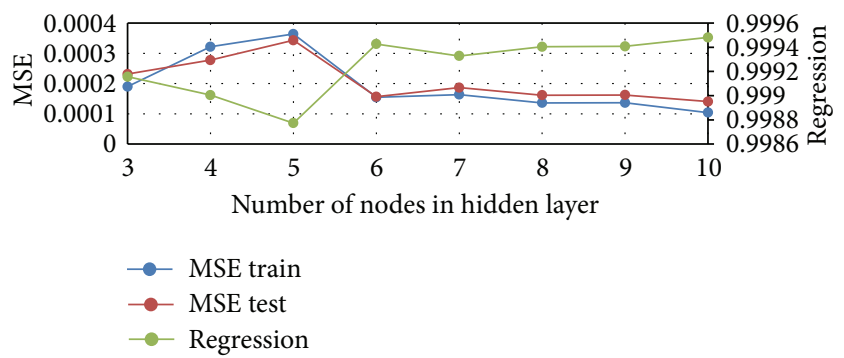

FIGURE 4: MSE with the number of nodes in hidden layer.

4.2. GRNN Method. GRNN as a probabilistic model can perform classification and regression for definite and continuous targets [8]. For the purpose of normalization, (6) and (7) are used in this model with some differences.

The values $a_{\max }=1$ and $a_{\min }=0$ are applied in (6) and (7). The error calculation is the same as in Section 4.1 and (8).

\section{Result and Discussion}

A trial for investigation of the number of nodes in the hidden layer is evaluated to find the MSE of the FFBP neural network. Table 2 represents the training MSE, testing MSE, and regression value for different numbers of hidden nodes for FFBP. In addition, a graphical visualization of MSE and regression based on the number of nodes in the hidden layer is shown in Figure 4.

The comparison between the simulated results of the FFBP and the real data for the power of the solar panel in 2010 is provided in Figures 5, 6, and 7. These figures illustrate these comparisons for January, February, and March of 2010.

The results for these three months demonstrate that the results are accurate for the FFBP model. The hidden neurons and the number of epochs in this calculation are 10 and 1000 , respectively. The same comparison for one year (2010) is provided in Figure 8 with six hidden neurons and 1000 epochs.

Figure 8 shows that the differences of predicted power and real power are very low and a high level of accuracy is achieved by using FFBP. A number of hidden neurons and the number of epochs are changed to examine the result as shown in Figure 9. 


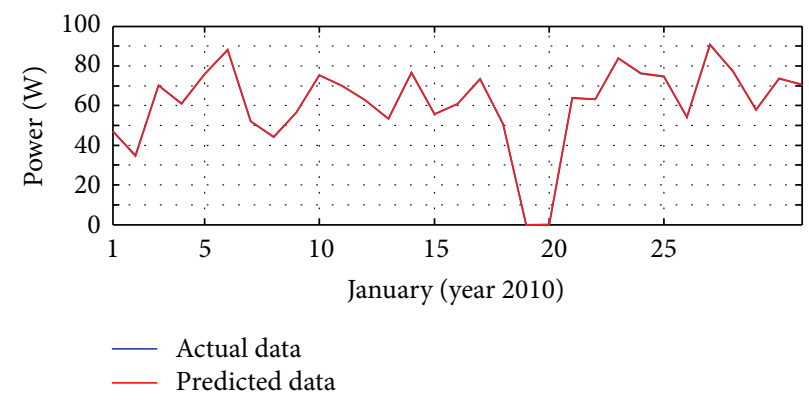

FIGURE 5: Results for real data and FFBP for January 2010.

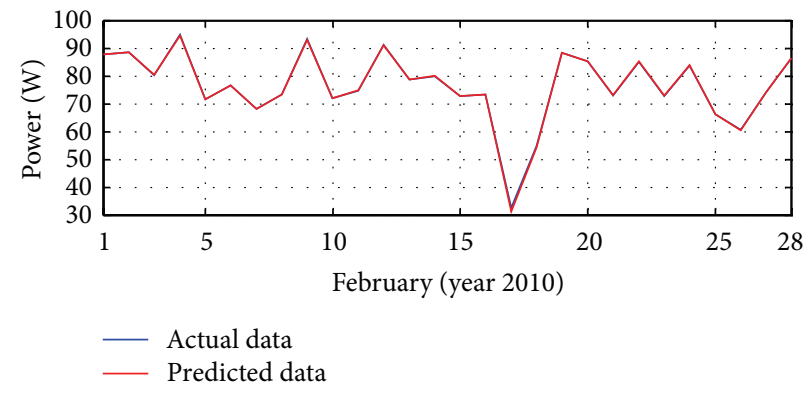

FIgURE 6: Results for real data and FFBP for February 2010.

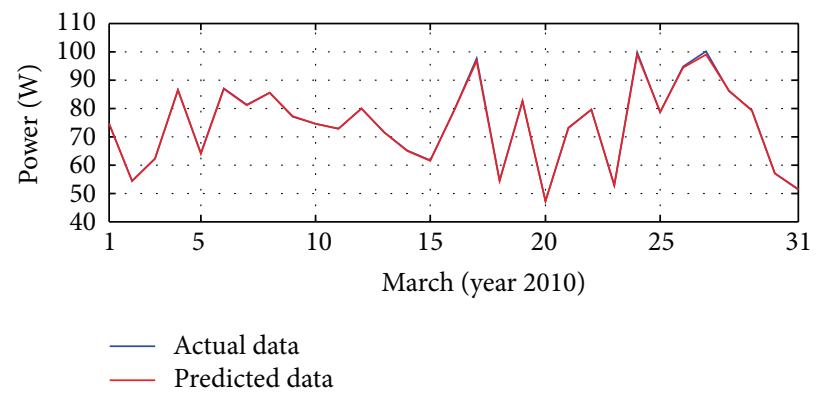

FIgURE 7: Results for real data and FFBP for March 2010.

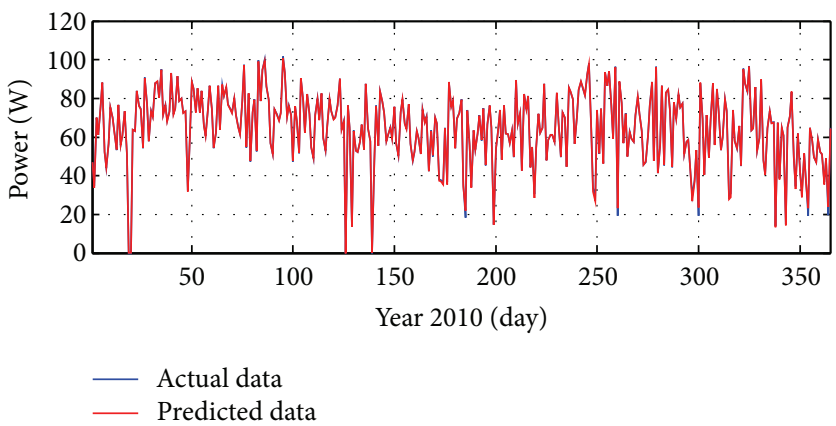

FIgURE 8: Results for real data and FFBP for 2010 with 6 hidden neurons and 1000 epochs.

Although the number of hidden layers is increased from 6 hidden neurons to 10 hidden neurons in Figure 9, the level of accuracy is very close by considering Figures 8 and 9 . Figure 10 presents the regression of calculating data and the real data. As shown in this figure, the measured error is very low.

According to Figure 11 the best validation performance is $4.4514 \times 10^{-7}$ at epoch 199 . 


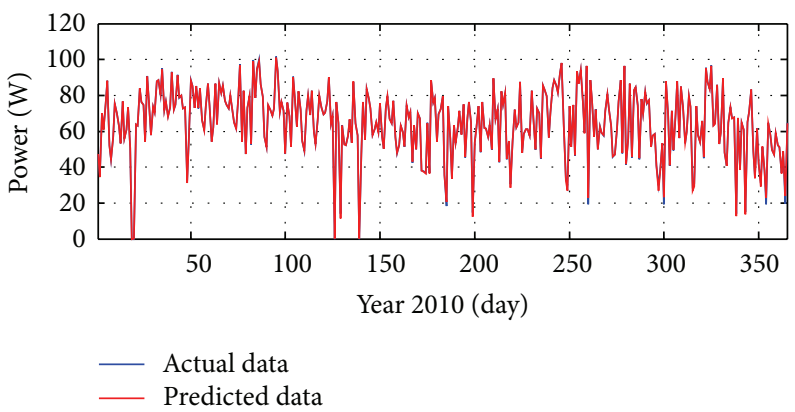

FIGURE 9: Results for real data and FFBP for 2010 with 10 hidden neurons.

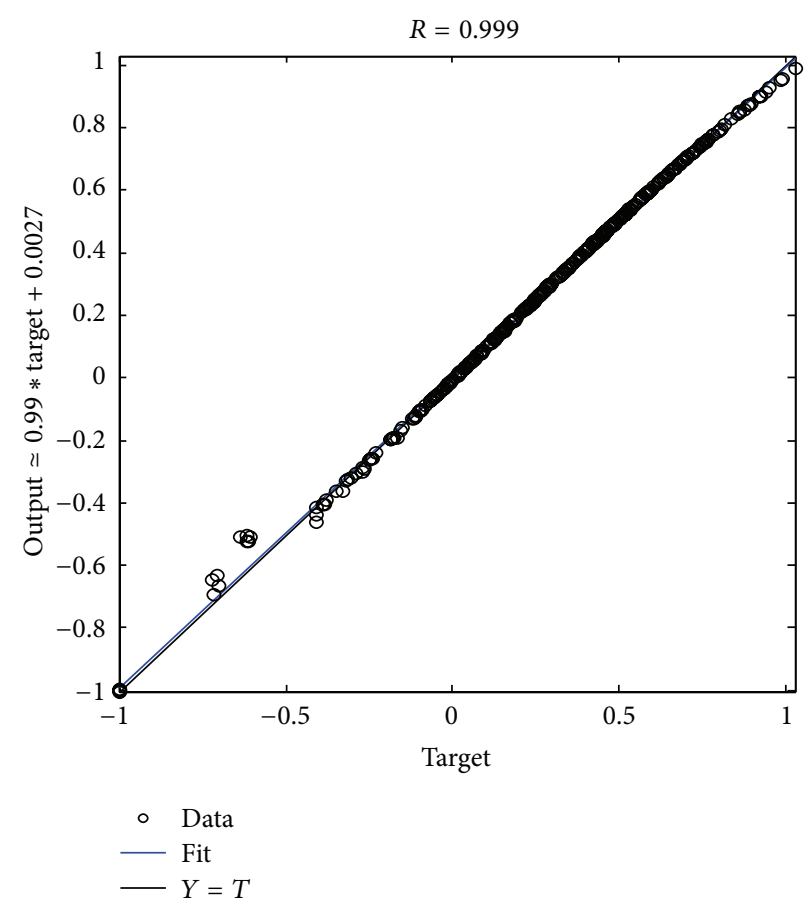

FIGURE 10: FFBP regression of error.

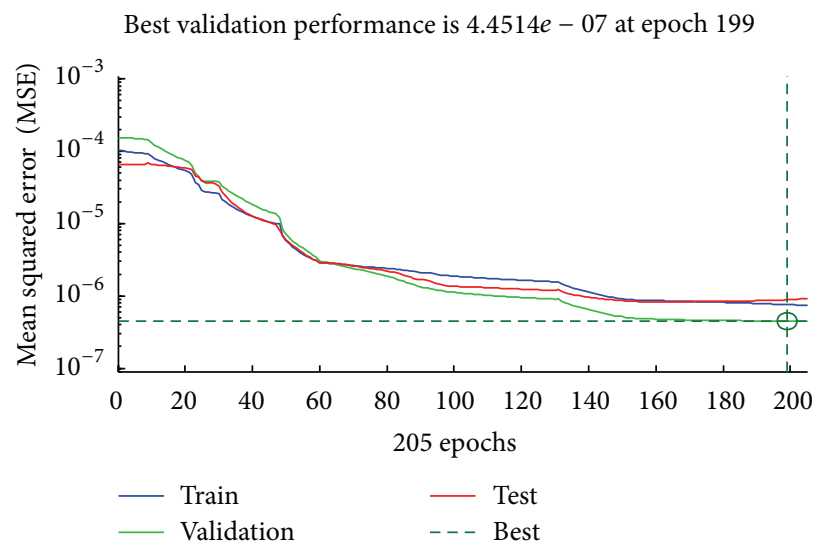

FIGURE 11: The training performance for FFBP with 10 neurons. 


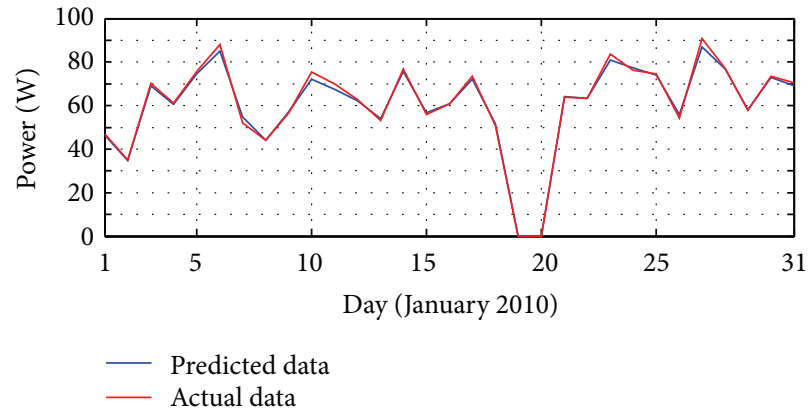

FIGURE 12: Results of real data and GRNN for January 2010.

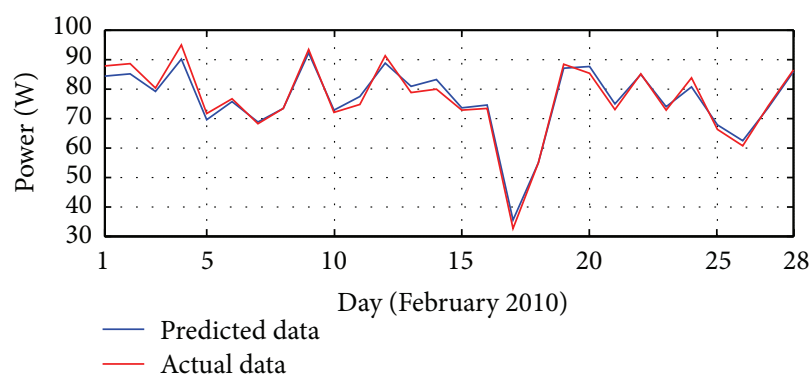

FIGURE 13: Results of real data and GRNN for February 2010.

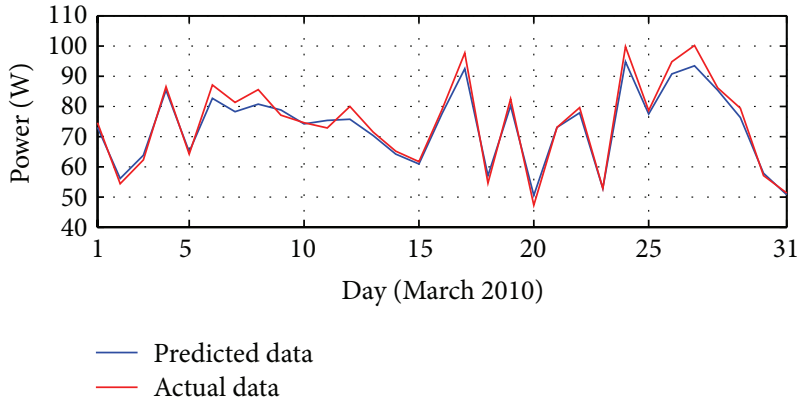

FIGURE 14: Results of real data and GRNN for March 2010.

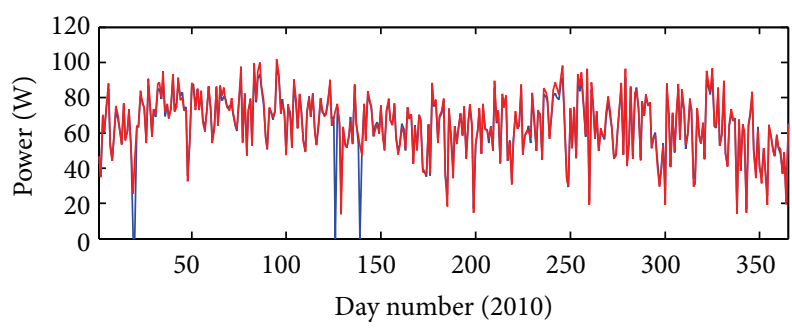

- Predicted data

FIGURE 15: Overall results for real data and GRNN for 2010.

GRNN network has only one free parameter as spread which is the distance of an input from a neuron's weight [40]. Different spread values have been examined to find the best

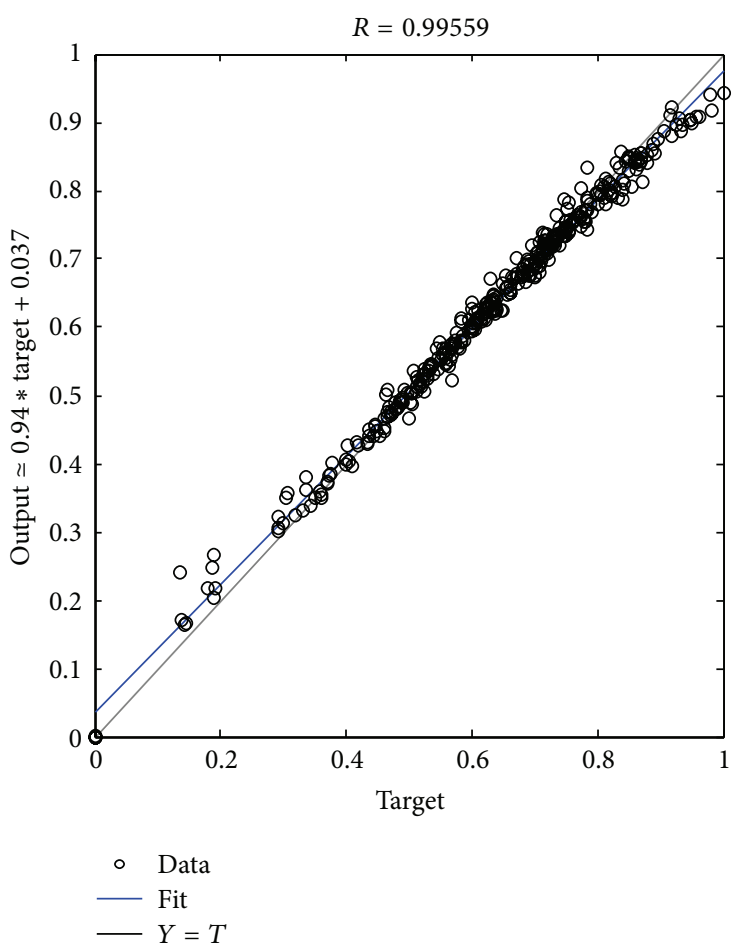

FIGURE 16: Comparison between measured and predicted data.

fit as shown in Table 3 . The monthly measured testing data are presented in Figures 12, 13, and 14 based on a spread of 0.04 .

Comparing the results of FFBP and GRNN shows that FFBP is more accurate compared with GRNN. 
TABLE 3: The GRNN comparison for different spreads.

\begin{tabular}{lccc}
\hline Spread & MSE train & MSE test & Regression \\
\hline 0.01 & $5.16 \times 10^{-8}$ & 0.00072 & 0.98948 \\
0.02 & $4.15 \times 10^{-6}$ & 0.00054 & 0.99238 \\
0.03 & $2.23 \times 10^{-5}$ & 0.0004 & 0.99476 \\
0.04 & $6.80 \times 10^{-5}$ & 0.00039 & 0.99559 \\
0.05 & 0.00015 & 0.00049 & 0.99505 \\
0.06 & 0.00029 & 0.00068 & 0.99362 \\
0.07 & 0.00048 & 0.00094 & 0.99151 \\
0.08 & 0.00072 & 0.00126 & 0.98882 \\
0.09 & 0.001 & 0.00162 & 0.98575 \\
0.1 & 0.00131 & 0.00201 & 0.98243 \\
\hline
\end{tabular}

TABLE 4: Sample data for modelling of PV.

\begin{tabular}{|c|c|c|c|c|c|c|c|}
\hline Year & Month & Day & $N$ & Total solar KW/m² & $V_{\text {oc }}$ at IR at $25^{\circ} \mathrm{C}$ & $V_{\text {mpp }}$ at IR at Max temp. & Output power \\
\hline 2006 & 1 & 1 & 1 & 0.274841223 & 27.181649 & 0.058270974 & 42.6518 \\
\hline 2006 & 1 & 2 & 2 & 0.203071904 & 26.6355684 & 0.042189698 & 30.7373 \\
\hline 2006 & 1 & 3 & 3 & 0.384548966 & 27.8008342 & 0.0833881 & 61.5857 \\
\hline 2006 & 1 & 4 & 4 & 0.365065203 & 27.7040672 & 0.078887569 & 57.9466 \\
\hline 2006 & 1 & 5 & 5 & 0.352615893 & 27.6396815 & 0.07602029 & 55.6912 \\
\hline 2006 & 1 & 6 & 6 & 0.299843763 & 27.3408209 & 0.063944202 & 46.9139 \\
\hline 2006 & 1 & 7 & 7 & 0.197615363 & 26.5869615 & 0.040981138 & 29.8238 \\
\hline 2006 & 1 & 8 & 8 & 0.121888679 & 25.7392373 & 0.024471109 & 13.5845 \\
\hline 2006 & 1 & 9 & 9 & 0.175552495 & 26.376727 & 0.036117902 & 18.5403 \\
\hline 2006 & 1 & 10 & 10 & 0.255924153 & 27.051971 & 0.054001371 & 39.564 \\
\hline 2006 & 1 & 11 & 11 & 0.252152139 & 27.0250476 & 0.053152504 & 38.9175 \\
\hline 2006 & 1 & 12 & 12 & 0.301336286 & 27.3499262 & 0.064283897 & 47.1211 \\
\hline 2006 & 1 & 13 & 13 & 0.412130472 & 27.9302742 & 0.089785153 & 66.1377 \\
\hline 2006 & 1 & 14 & 14 & 0.474875137 & 28.1969664 & 0.104442299 & 76.9622 \\
\hline 2006 & 1 & 15 & 15 & 0.198878834 & 26.5983269 & 0.041260785 & 30.0045 \\
\hline 2006 & 1 & 16 & 16 & 0.400291667 & 27.8757359 & 0.087035713 & 64.3705 \\
\hline 2006 & 1 & 17 & 17 & 0.283381772 & 27.2374867 & 0.060205136 & 44.1317 \\
\hline 2006 & 1 & 18 & 18 & 0.395752595 & 27.8544257 & 0.085982998 & 63.4632 \\
\hline 2006 & 1 & 19 & 19 & 0.417949979 & 27.9565494 & 0.091138626 & 66.9412 \\
\hline 2006 & 1 & 20 & 20 & 0.425391254 & 27.9896541 & 0.092871122 & 68.2328 \\
\hline 2006 & 1 & 21 & 21 & 0.335214455 & 27.5460362 & 0.07202387 & 52.8023 \\
\hline 2006 & 1 & 22 & 22 & 0.393920428 & 27.8457593 & 0.085558305 & 62.935 \\
\hline 2006 & 1 & 23 & 23 & 0.325532825 & 27.491952 & 0.069806356 & 51.3257 \\
\hline 2006 & 1 & 24 & 24 & 0.287815825 & 27.26586 & 0.061210859 & 44.8429 \\
\hline 2006 & 1 & 25 & 25 & 0.429558971 & 28.0079601 & 0.09384235 & 69.3494 \\
\hline 2006 & 1 & 26 & 26 & 0.430434611 & 28.0117851 & 0.094046486 & 69.4158 \\
\hline 2006 & 1 & 27 & 27 & 0.37166462 & 27.737372 & 0.080410199 & 59.241 \\
\hline 2006 & 1 & 28 & 28 & 0.468196434 & 28.1701967 & 0.102875648 & 75.6425 \\
\hline 2006 & 1 & 29 & 29 & 0.438430519 & 28.0463815 & 0.095911839 & 70.5903 \\
\hline 2006 & 1 & 30 & 30 & 0.356302403 & 27.6589657 & 0.076868656 & 56.5022 \\
\hline 2006 & 1 & 31 & 31 & 0.413284534 & 27.9355122 & 0.090053458 & 66.2356 \\
\hline
\end{tabular}

Figure 15 demonstrates that the applied methodology and process have been right. There are a few changes between measured and predicted power. The prediction of power using FFBP was more accurate compared to GRNN. Figure 16 provides information about variation of measured data and predicted values. The predicted values are around the real one and show the level of accuracy.

\section{Conclusion}

Power prediction for photovoltaic panels is needed for accurate power planning. In this paper, the generated power of a solar panel has been estimated using mathematical equations. Afterward, the meteorological data and estimated power have been used for training GRNN and FFBP. Both of these neural 
networks have shown good modelling performance; however, FFBP has shown a better performance compared to GRNN.

\section{Conflict of Interests}

The authors declare no conflict of interests regarding the publication of this paper.

\section{References}

[1] L. Martín, L. F. Zarzalejo, J. Polo, A. Navarro, R. Marchante, and M. Cony, "Prediction of global solar irradiance based on time series analysis: application to solar thermal power plants energy production planning," Solar Energy, vol. 84, no. 10, pp. 17721781, 2010.

[2] D. Heinemann, E. Lorenz, and M. Girodo, "Forecasting of solar radiation," in Solar Energy Resource Management for Electricity Generation From Local Level to Global Scale, Nova Science Publishers, New York, NY, USA, 2006.

[3] R. Perez, K. Moore, S. Wilcox, D. Renné, and A. Zelenka, "Forecasting solar radiation-preliminary evaluation of an approach based upon the national forecast database," Solar Energy, vol. 81, no. 6, pp. 809-812, 2007.

[4] A. Mellit and A. M. Pavan, "A 24-h forecast of solar irradiance using artificial neural network: application for performance prediction of a grid-connected PV plant at Trieste, Italy," Solar Energy, vol. 84, no. 5, pp. 807-821, 2010.

[5] Z. Wang, F. Wang, and S. Su, "Solar irradiance short-term prediction model based on BP neural network," Energy Procedia, vol. 12, pp. 488-494, 2011.

[6] K. Hornik, M. Stinchcombe, and H. White, "Multilayer feedforward networks are universal approximators," Neural Networks, vol. 2, no. 5, pp. 359-366, 1989.

[7] W. S. McCulloch and W. Pitts, "A logical calculus of the ideas immanent in nervous activity," The Bulletin of Mathematical Biophysics, vol. 5, no. 4, pp. 115-133, 1943.

[8] T. Khatib, A. Mohamed, K. Sopian, and M. Mahmoud, "Assessment of artificial neural networks for hourly solar radiation prediction," International Journal of Photoenergy, vol. 2012, Article ID 946890, 7 pages, 2012.

[9] S. Krauter and T. Depping, "Monitoring of remote PV-systems via satellite," in Proceddings of the 3rd World Conference on Photovoltaic Energy Conversion, pp. 2202-2205, May 2003.

[10] J. D. Mondol, Y. G. Yohanis, and B. Norton, “The effect of low insolation conditions and inverter oversizing on the long-term performance of a grid-connected photovoltaic system," Progress in Photovoltaics: Research and Applications, vol. 15, no. 4, pp. 353-368, 2007.

[11] I. Morsy, A. Aboul Seoud, and A. El Zawawi, "On-line prediction of photovoltaic output power under cloudy skies by using fuzzy logic," in Proceedings of the 19th National Radio Science Conference (NRSC '02), pp. 519-526.

[12] A. V. Timbus, R. Teodorescu, F. Blaabjerg, and U. Borup, "Online grid measurement and ENS detection for PV inverter running on highly inductive grid," IEEE Power Electronics Letters, vol. 2, no. 3, pp. 77-82, 2004.

[13] E. E. van Dyk, E. L. Meyer, F. J. Vorster, and A. W. R. Leitch, "Long-term monitoring of photovoltaic devices," Renewable Energy, vol. 25, no. 2, pp. 183-197, 2002.

[14] R. Mantri, H. Mangalvedhekar, and P. Gupta, "Weather sensitive short term load forecasting using fully connected feed forward neural network," International Journal of Engineering, vol. 2, 2013.

[15] K. A. Kumari, N. K. Boiroju, T. Ganesh, and P. R. Reddy, "Forecasting surface air temperature using neural networks," International Journal of Mathematics and Computer Applications Research, vol. 3, pp. 65-78, 2012.

[16] A. Mellit and S. A. Kalogirou, "Artificial intelligence techniques for photovoltaic applications: a review," Progress in Energy and Combustion Science, vol. 34, no. 5, pp. 574-632, 2008.

[17] M. A. AbdulAzeez, "Artificial neural network estimation of global solar radiation using meteorological parameters in Gusau, Nigeria," Archives of Applied Science Research, vol. 3, pp. 586-595, 2011.

[18] C. Chen, S. Duan, T. Cai, and B. Liu, "Online 24-h solar power forecasting based on weather type classification using artificial neural network," Solar Energy, vol. 85, no. 11, pp. 2856-2870, 2011.

[19] F. O. Hocaoğlu, Ö. N. Gerek, and M. Kurban, "Hourly solar radiation forecasting using optimal coefficient 2-D linear filters and feed-forward neural networks," Solar Energy, vol. 82, no. 8, pp. 714-726, 2008.

[20] A. Linares-Rodríguez, J. A. Ruiz-Arias, D. Pozo-Vázquez, and J. Tovar-Pescador, "Generation of synthetic daily global solar radiation data based on ERA-Interim reanalysis and artificial neural networks," Energy, vol. 36, no. 8, pp. 5356-5365, 2011.

[21] C. Paoli, C. Voyant, M. Muselli, and M.-L. Nivet, "Forecasting of preprocessed daily solar radiation time series using neural networks," Solar Energy, vol. 84, no. 12, pp. 2146-2160, 2010.

[22] W. Chen and S. Varadarajan, "Integration of design of experiments and artificial neural networks for achieving affordable concurrent design," in Proceedings of the 38th AIAA/ASME/ ASCE/AHS/ASC Structures, Structural Dynamics, and Materials Conference and AIAA/ASME/AHS Adaptive Structures Forum, pp. 1316-1324, April 1997.

[23] A. Mellit and S. Shaari, "Recurrent neural network-based forecasting of the daily electricity generation of a Photovoltaic power system," in Ecological Vehicle and Renewable Energy (EVER), pp. 26-29, Monte-Carlo, Monaco, March 2009.

[24] E. A. Ahmed and M. E.-N. Adam, "Estimate of global solar radiation by using Artificial Neural Network in Qena, Upper Egypt," Journal of Clean Energy Technologies, vol. 1, no. 2, pp. 148-150, 2013.

[25] S. Naini, "Evaluation of RBF, GR and FFBP neural networks for prediction of geometrical dimensions of scour hole below ski-jump spillway," in Proceedings of the IEEE International Conference on Electronics, Circuits, and Systems (ICECS '11), vol. 19, pp. 89-93, IACSIT Press, Singapore, 2011.

[26] T. Vigneswaran and S. Dhivya, "Analyzing the probabilistic distribution of the predicted wind speed," International Journal of Computer and Information Technology, vol. 1, pp. 88-93, 2012.

[27] K. Upadhyay, A. Choudhary, and M. Tripathi, "Short-term wind speed forecasting using feed-forward back-propagation neural network," International Journal of Engineering, Science and Technology, vol. 3, no. 5, pp. 107-112, 2011.

[28] F. A. Makinde, C. T. Ako, O. D. Orodu, and I. U. Asuquo, "Prediction of crude oil viscosity using feed-forward backpropagation neural network (FFBPNN)," Petroleum and Coal, vol. 54, pp. 120-131, 2012.

[29] C. J. Devi, B. S. P. Reddy, K. V. Kumar, B. M. Reddy, and N. Raja, "ANN approach for weather prediction using back propagation," International Journal of Engineering Trends and Technology, vol. 3, no. 1, 2012. 
[30] K. O. Alawode and M. O. Oyedeji, "A comparison of neural network models for load forecasting in nigerian power system," International Journal of Research in Engineering and Technology, vol. 2, no. 5, 2013.

[31] T. Krishnaiah, S. Srinivasa Rao, K. Madhumurthy, and K. Reddy, "Neural network approach for modelling global solar radiation," Journal of Applied Sciences Research, vol. 3, pp. 1105-1111, 2007.

[32] S. Zhao, J. Zhao, G. Zhao, W. Zhang, and Z. Guo, "Effective wind power density prediction based on neural networks," in Proceedings of the International Conference on Multimedia Technology (ICMT '10), pp. 1-4, October 2010.

[33] P. M. Gotovtsev, D. S. Smetanin, and V. N. Voronov, "Prediction of water chemistry state by means of artificial neural network," in International Conference on the Properties of Water and Steam, pp. 1-3, September 2008.

[34] G. Corani, "Air quality prediction in Milan: feed-forward neural networks, pruned neural networks and lazy learning," Ecological Modelling, vol. 185, no. 2-4, pp. 513-529, 2005.

[35] T. Khatib, A. Mohamed, K. Sopian, and M. Mahmoud, "Solar energy prediction for Malaysia using artificial neural networks," International Journal of Photoenergy, vol. 2012, Article ID 419504, 16 pages, 2012.

[36] D. F. Specht, "A general regression neural network," IEEE Transactions on Neural Networks, vol. 2, no. 6, pp. 568-576, 1991.

[37] C. Budischak, D. Sewell, H. Thomson, L. Mach, D. E. Veron, and W. Kempton, "Cost-minimized combinations of wind power, solar power and electrochemical storage, powering the grid up to $99.9 \%$ of the time," Journal of Power Sources, vol. 225, pp. 6074, 2013.

[38] Sharp data sheet, 2007, http://www.sharpcentrum.com/filead$\mathrm{min} /$ dokumenty/solar_panely/Sharp_NU-S0E3E_NU-180_E1_180Wp_NU-S5E3E_NU-185_E1__185Wp_EN.pdf.

[39] T. Jayalakshmi and A. Santhakumaran, "Statistical normalization and back propagation for classification," International Journal of Computer Theory and Engineering, vol. 3, pp. 17938201, 2011.

[40] I. Popescu, P. Constantinou, M. Nafornita, and L. Nafornita, "Generalized Regression Neural Network prediction model for indoor environment," in Proceedings of the 9th International Symposium on Computers and Communications (ISCC '04), pp. 657-661, July 2004. 

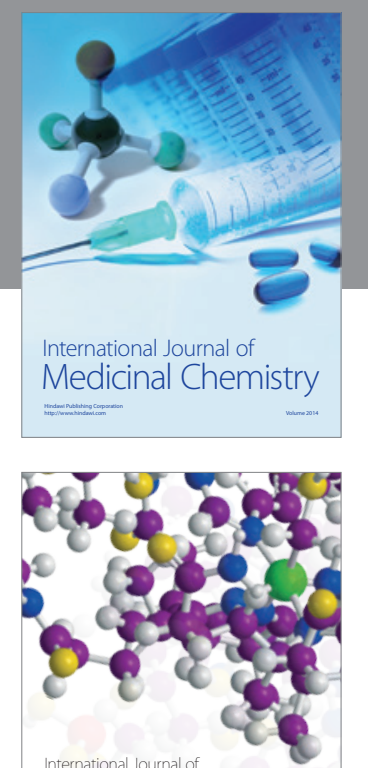

\section{Carbohydrate} Chemistry

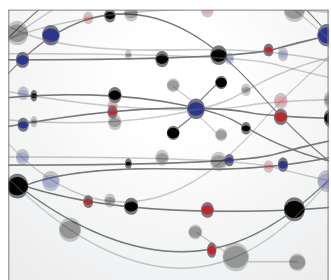

The Scientific World Journal
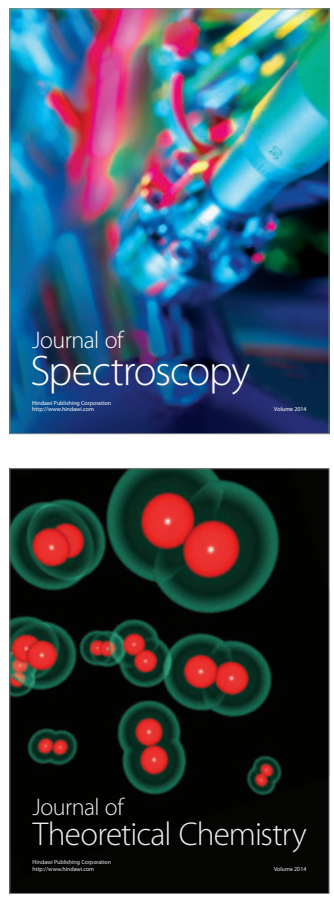
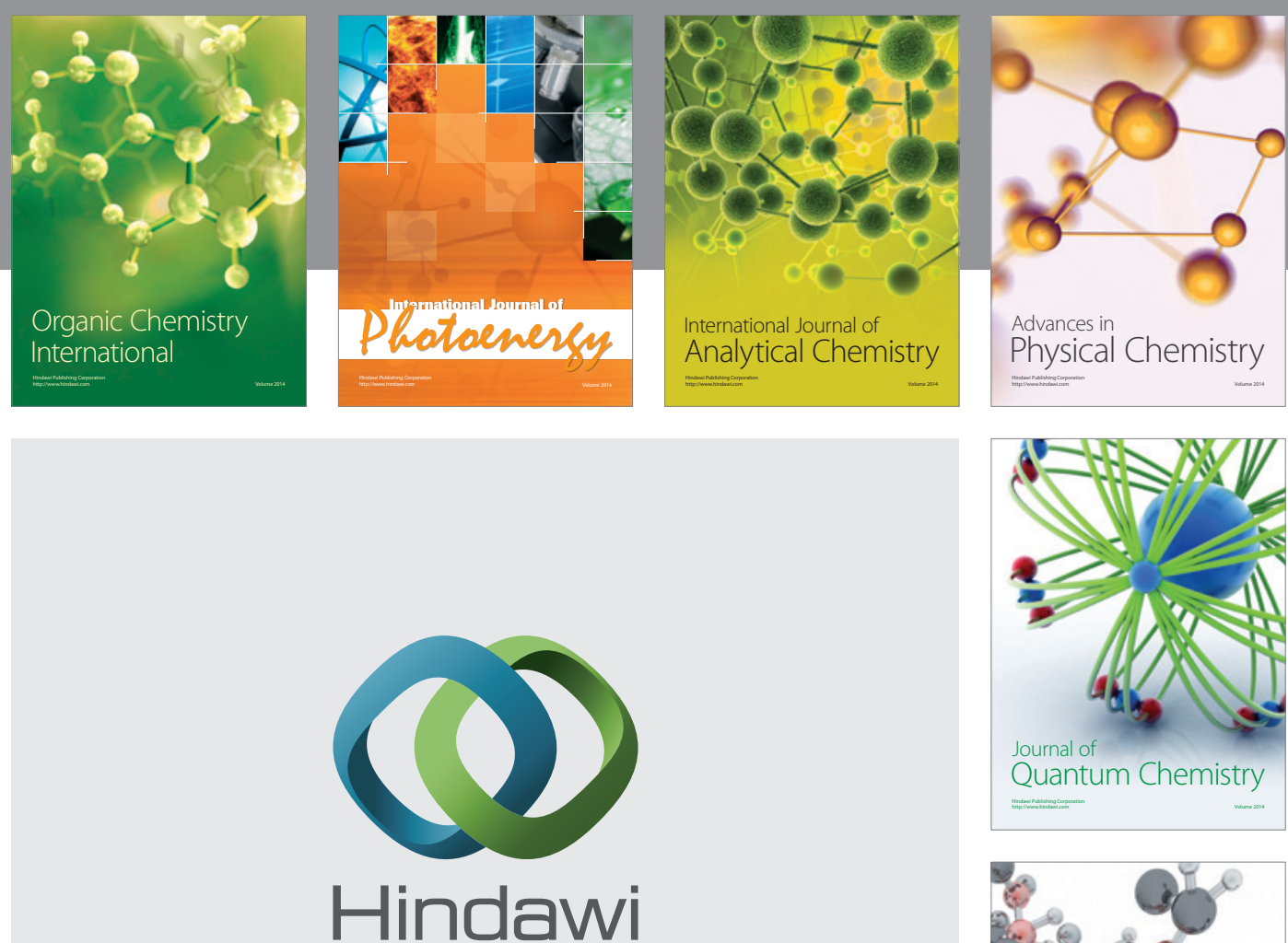

Submit your manuscripts at

http://www.hindawi.com

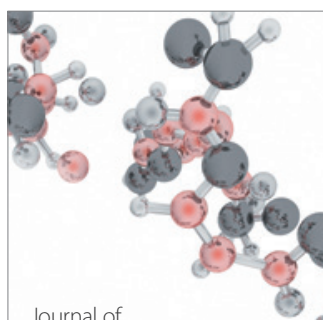

Analytical Methods

in Chemistry

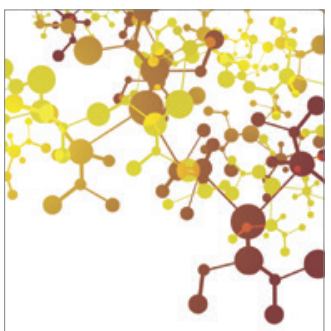

Journal of

Applied Chemistry

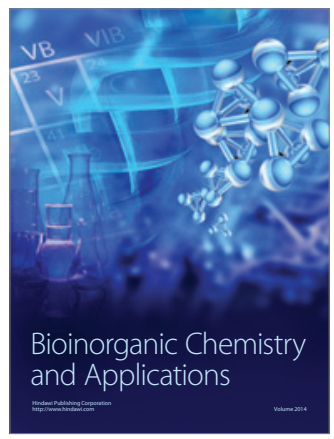

Inorganic Chemistry
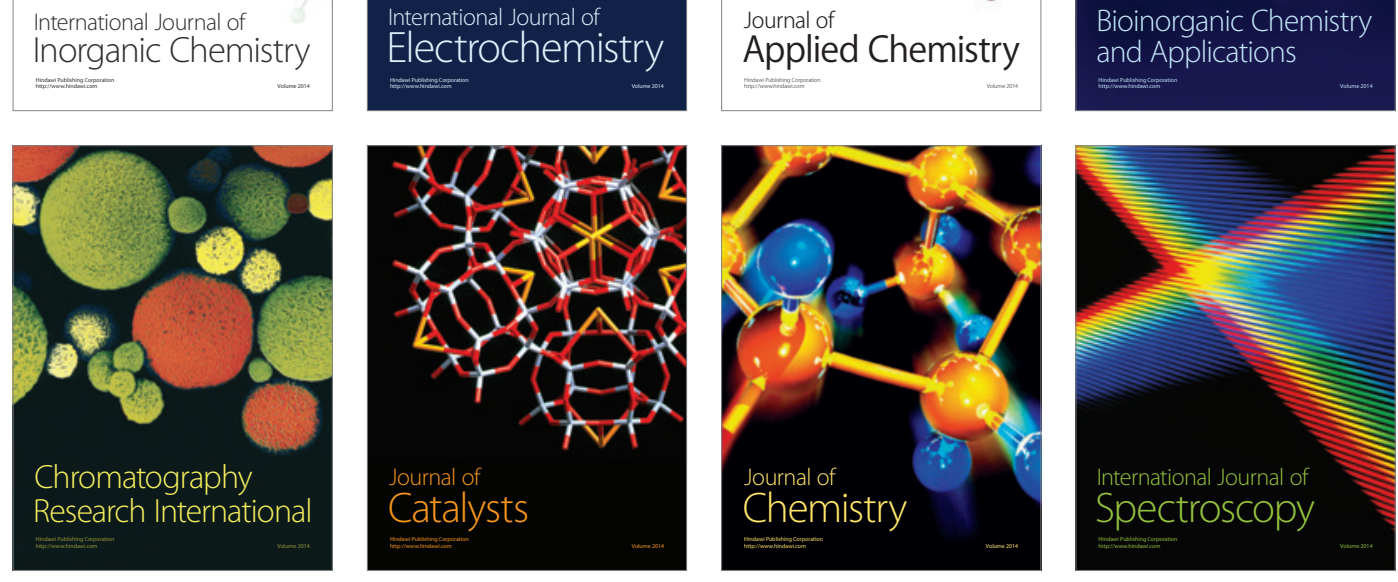